\title{
Hyperbaric oxygen therapy for the treatment of long COVID: early evaluation of a highly promising intervention
}

\author{
Authors: Tim Robbins, ${ }^{\mathrm{A}}$ Michael Gonevski, ${ }^{\mathrm{B}}$ Cain Clark, ${ }^{\mathrm{C}}$ Sudhanshu Baitule, ${ }^{\mathrm{D}}$ Kavi Sharma, ${ }^{\mathrm{E}}$ Angel Magar, ${ }^{\mathrm{F}}$ \\ Kiran Patel, ${ }^{\mathrm{G}}$ Sailesh Sankar, ${ }^{\mathrm{H}}$ Ioannis Kyrou, ${ }^{\mathrm{I}}$ Asad Ali ${ }^{3}$ and Harpal S Randeva ${ }^{\mathrm{K}}$
}

\section{Background}

Long COVID is a common occurrence following COVID-19 infection. The most common symptom reported is fatigue. Limited interventional treatment options exist. We report the first evaluation of hyperbaric oxygen therapy (HBOT) for long COVID treatment.

\section{Methods}

A total of 10 consecutive patients received 10 sessions of HBOT to 2.4 atmospheres over 12 days. Each treatment session lasted 105 minutes, consisting of three 30-minute exposures to $100 \%$ oxygen, interspersed with 5 -minute air breaks. Validated fatigue and cognitive scoring assessments were performed at day 1 and 10 . Statistical analysis was with Wilcoxon signed-rank testing reported alongside effect sizes.

Results

HBOT yielded a statistically significant improvement in the Chalder fatigue scale $(p=0.0059 ; d=1.75$ (very large)), global

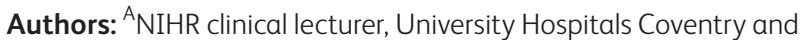
Warwickshire NHS Trust, Coventry, UK, Warwick Medical School, Coventry, UK and Coventry University, Coventry, UK; Bhyperbaric

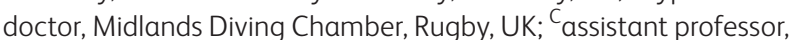
University Hospitals Coventry and Warwickshire NHS Trust, Coventry, UK and Coventry University, Coventry, UK; ${ }^{\mathrm{D}}$ Clinical fellow, University Hospitals Coventry and Warwickshire NHS Trust, Coventry, UK; Etrial manager, University Hospitals Coventry and Warwickshire NHS Trust, Coventry, UK; F research grant coordinator, University Hospitals Coventry and Warwickshire NHS Trust, Coventry, UK; ${ }^{G}$ chief medical officer, deputy chief executive officer and consultant cardiologist, University Hospitals Coventry and Warwickshire NHS Trust, Coventry, UK and Warwick Medical School, Coventry, UK; ${ }^{\mathrm{H}}$ associate medical director, University Hospitals Coventry and Warwickshire NHS Trust, Coventry, UK

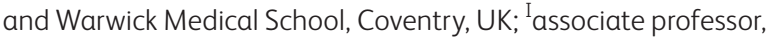
University Hospitals Coventry and Warwickshire NHS Trust, Coventry, UK, Warwick Medical School, Coventry, UK and Coventry University, Coventry, UK; ' consultant sleep and respiratory physician, University Hospitals Coventry and Warwickshire NHS Trust, Coventry, UK and Warwick Medical School, Coventry, UK; ${ }^{K}$ director of research and development, University Hospitals Coventry and Warwickshire NHS Trust, Coventry, UK and Warwick Medical School, Coventry, UK cognition ( $p=0.0137 ; d=-1.07$ (large)), executive function $(p=0.0039 ; d=-1.06$ (large)), attention $(p=0.0020 ; d=-1.2$ (very large)), information processing $(p=0.0059 ; d=-1.25$ (very large)) and verbal function ( $\mathrm{p}=0.0098 ; \mathrm{d}=-0.92$ (large)).

\section{Conclusion}

Long COVID-related fatigue can be debilitating, and may affect young people who were previously in economic employment. The results presented here suggest potential benefits of $\mathrm{HBOT}$, with statistically significant results following 10 sessions.

KEYWORDS: long COVID, hyperbaric oxygen therapy, fatigue

DOI: $10.7861 /$ clinmed.2021-0462

\section{Introduction}

The COVID-19 pandemic has resulted in the need to support large cohorts of patients suffering from long COVID after recovery from acute infection. 'Long COVID is a term 'used to describe presence of various symptoms, even weeks or months after acquiring SARS-CoV-2 infection irrespective of the viral status'. ${ }^{2}$ Long COVID is a common condition, with estimates identifying that between $10 \%$ and $20 \%$ of people initially diagnosed with acute COVID-19 will go on to develop symptoms of long COVID. ${ }^{3}$ There remains some debate about the terminology used in long COVID, with the UK National Institute for Health and Care Excellence (NICE) also using the terminology 'post-COVID-19 syndrome' for signs and symptoms that develop during or after an infection consistent with COVID-19, continue for more than 12 weeks and are not explained by an alternative diagnosis, and 'ongoing symptomatic COVID-19' for signs and symptoms of COVID-19 from 4 to 12 weeks. $^{3}$ However, NICE also recognises the umbrella term 'long COVID'. Long COVID now poses an emerging public health emergency with multiple challenges for the management of these patients in clinical practice. ${ }^{4-6}$ The symptoms associated with long COVID are diverse, including breathlessness, cough, fatigue, 'brain fog', anxiety and depression. ${ }^{7}$ One of the most commonly reported symptoms is fatigue, present in up to $65 \%$ of long COVID patients, this is accompanied by a substantial proportion also reporting cognitive and affective deficits (described in the literature as 'brain fog'). ${ }^{8-11}$ The fatigue experienced by these patients can be particularly severe, preventing them from performing their usual 
work and activities, while the age group most affected by long COVID tends to be economically active adults. ${ }^{12}$

Currently specific treatment options for long COVID are limited, with even fewer treatment options available for those suffering from fatigue. ${ }^{13,14}$ Thus, there is a growing need to identify effective treatments for these patients. ${ }^{15}$ Despite distinct differences, there are important similarities between long COVID fatigue and chronic fatigue syndrome. ${ }^{9,16}$

Hyperbaric oxygen therapy (HBOT) is "an intervention where an individual breathes near $100 \%$ oxygen intermittently while inside a hyperbaric chamber that is pressurized to greater than sea level pressure (1 atmosphere absolute, or ATA), ${ }^{17,18}$ HBOT is used for both elective (eg soft tissue radiation complications and non-healing chronic wounds) and emergency medical conditions (eg carbon monoxide poisoning, decompression illness and gas embolism). ${ }^{17}$ In particular, HBOT has been shown to be safe and effective in the treatment of chronic fatigue syndrome. ${ }^{19,20}$ At University Hospitals Coventry and Warwickshire NHS Trust, working in direct partnership with the Midlands Diving Chamber at the Rugby Hospital of St Cross, we proposed that HBOT may be of benefit to people suffering from symptoms of long COVID. The first patient to receive HBOT for long COVID received this on the 11 January 2021. Currently, there is no study to our knowledge which explored the effects of HBOT on long COVID-related fatigue. Here we present the first evaluation of a HBOT service for the treatment of long COVID symptoms internationally.

\section{Methods}

We retrospectively evaluated the response of fatigue symptoms of patients with long COVID-related fatigue receiving HBOT at the Midlands Diving Chamber medical facility, Hospital of St Cross, Rugby. This retrospective evaluation was approved by the University Hospitals Coventry and Warwickshire NHS Trust COVID-19 Research Ethics Committee through the GAFREC Process (ID: 10026).

We evaluated 10 consecutive patients undergoing HBOT for long COVID-related fatigue at the Midlands Diving Chamber. All patients were suffering from new fatigue that developed during or after an infection consistent with COVID-19 and continuing for more than 12 weeks. The clinical inclusion and exclusion criteria for these patients are listed in Table 1.

All patients received 10 HBOT sessions, once daily at 2.4 atmospheres for 1 hour and 45 minutes over 12 days (with a 2-day break in the middle for the weekend).

The responses of these 10 consecutive patients receiving HBOT therapy were evaluated with the primary outcome measure being the change in Chalder fatigue scale between days 1 and 10 of treatment. ${ }^{21}$ The secondary measure evaluated was the change in the cognitive profile scores of day 1 and day 10 , as reported through the NeuroTrax evaluation. ${ }^{22}$ This scoring included global cognitive score, memory, executive function, attention, information processing speed, visual-spatial, verbal function and motor skills.

\section{Statistical analysis}

In order investigate differences between day 1 and day 10 undergoing HBOT, Wilcoxon signed-rank testing was conducted, and reported alongside corresponding effect sizes (Cohen's d; classified as small (0.2), medium (0.5), large (0.8) or very large (1.2),
Table 1. Inclusion and exclusion criteria for

hyperbaric oxygen therapy

\begin{tabular}{|c|c|}
\hline Inclusion criteria & Exclusion criteria \\
\hline $\begin{array}{l}\text { Age above } 18 \text { years } \\
\text { Previous confirmed } \\
\text { COVID-19 infection } \\
\text { diagnosed with } \\
\text { swab PCR test or } \\
\text { positive antibody } \\
\text { test }\end{array}$ & $\begin{array}{l}\text { History of traumatic brain injury } \\
\text { or any other non-COVID brain } \\
\text { pathology } \\
\text { > Active malignancy (current solid } \\
\text { organ or blood cancer either under } \\
\text { active treatment, observation or } \\
\text { palliative care). }\end{array}$ \\
\hline $\begin{array}{l}\text { Subject willing } \\
\text { and able to read, } \\
\text { understand and } \\
\text { sign an informed } \\
\text { consent to have } \\
\text { hyperbaric oxygen } \\
\text { therapy }\end{array}$ & $\begin{array}{l}\text { Substance use at baseline (alcohol } \\
\text { use in excess of current government } \\
\text { guidelines) } \\
>\text { Severe or unstable physical disorders } \\
\text { or major cognitive deficits at baseline } \\
>\text { HBOT for any reason prior to study } \\
\text { enrolment }\end{array}$ \\
\hline $\begin{array}{l}\text { Patient suffering } \\
\text { severe, longstand- } \\
\text { ing post-COVID-19 } \\
\text { syndrome }\end{array}$ & $\begin{array}{l}\text { Chest pathology incompatible } \\
\text { with pressure changes (including } \\
\text { moderate to severe asthma, COPD } \\
\text { and history of pneumothorax) } \\
\text { > Epilepsy } \\
>\text { Ear or sinus pathology incompatible } \\
\text { with pressure changes } \\
\text { > Claustrophobia }\end{array}$ \\
\hline
\end{tabular}

and $95 \%$ confidence intervals (CIs)). ${ }^{23}$ In addition, Bayes factors were also calculated to express the probability of a difference given $\mathrm{H}_{10}$ (alternate hypothesis) relative to $\mathrm{H}_{01}$ (null hypothesis), that is, values larger than 1 are in favour of $\mathrm{H}_{10}$ assuming that $\mathrm{H}_{01}$ and $\mathrm{H}_{10}$ are equally likely and using a default prior. ${ }^{24}$ Bayes factors were reported as the probability of the data given the alternative relative to the null hypothesis or vice-versa (classified as anecdotal $\left({ }_{\mathrm{BF}} 1-3\right)$, moderate $\left.{ }_{\mathrm{BF}} 3-10\right)$, strong $\left.{ }_{\mathrm{BF}} 10-30\right)$, very strong $\left({ }_{\mathrm{BF}} 30-100\right)$ or extreme $\left.\left({ }_{\mathrm{BF}}>100\right)\right) .{ }^{25-27}$ Bayesian analysis was concurrently utilised because it permits the amalgamation of discipline-specific knowledge, facilitates direct probability statements to be made pertaining to included parameters (ie population level effects), allows zero effects to be determined, provides estimates of uncertainty around parameter values that are more intuitively interpretable than those from null hypothesis testing alone, and supports in the interpretation of $p$ values. ${ }^{28,29}$ All analyses were conducted using R software. ${ }^{30}$

\section{Results}

In the present cohort, $60 \%$ of the patients were women. The mean average age of participants was 47.5 years (range 24-74). All patients had been suffering from long COVID symptoms for over 3 months.

Participant level and overall group data for all collected validated scores between day 1 and day 10 of HBOT are presented in Table 2.

Wilcoxon signed-rank tests indicated that once daily HBOT for 10 days yielded a statistically significant improvement in Chalder fatigue scale $(p=0.0059 ; d=1.75$ (very large)), global cognition $(p=0.0137 ; d=-1.07$ (large)), executive function $(p=0.0039$; 
Table 2. Day 1 vs day 10 of hyperbaric oxygen therapy

\begin{tabular}{lllll} 
& p value & Mean difference (95\% CI) & Cohen's d & BF \\
Global cognition & $0.0137^{a}$ & $-8.4(-14.55--2.9)$ & -1.07 & 7.626 \\
Memory & 0.8457 & $0.9(-10.6-7)$ & -0.01 & 0.3091 \\
Executive function & $0.0039^{a}$ & $-7.3(-12.65--2.2)$ & -1.06 & 7.3286 \\
Attention & $0.0020^{a}$ & $-7(-12.45--2.05)$ & -1.2 & 12.5093 \\
IPS & $0.0059^{a}$ & $-15.3(-29.8--8.2)$ & -1.25 & 15.3199 \\
Visual-spatial & 0.1056 & $-5.5(-11.3-0.65)$ & -0.76 & 2.12 \\
Verbal function & $0.0098^{a}$ & $-21.95(-44.85--6.15)$ & -0.92 & 4.1335 \\
Motor skills & 0.0827 & $-3.9(-7.55-2.2)$ & -0.52 & 0.85 \\
Chalder fatigue scale & $0.0059^{a}$ & $18(9.5-26)$ & 1.75 & 98.13 \\
\hline${ }^{a}$ significant difference between time points; BF $=$ Bayes factor; CI = confidence interval; IPS = information processing speed.
\end{tabular}

$d=-1.06$ (large)), attention $(p=0.0020 ; d=-1.2$ (very large)), information processing speed ( $p=0.0059 ; d=-1.25$ (very large)) and verbal function $(p=0.0098 ; d=-0.92$ (large)). Concomitantly, Bayes factors indicated that the evidence favouring the alternative vs the null hypothesis was moderate for global cognition ( ${ }_{\mathrm{BF}} 7.63$ ), executive function ( ${ }_{\mathrm{BF}} 7.33$ ) and verbal function ( $\mathrm{BF}^{4} .13$ ); strong for attention ( ${ }_{B F} 12.51$ ) and information processing speed ( ${ }_{B F} 15.32$ ), and very strong for Chalder fatigue index (BF 98.13).

All pairwise comparisons including $p$ values, mean differences (95\% CIs), Cohen's d effect sizes and Bayes Factors are presented in Table 2 and Fig 1. Finally, participant level and overall group data for days 1 and 10 are presented in the supplementary material S1.

No adverse events were reported in any of these patients receiving $\mathrm{HBOT}$ during this treatment or in the immediate posttreatment phase.

\section{Discussion}

At University Hospitals Coventry and Warwickshire NHS Trust, we have established a dedicated clinician-led clinic for patients presenting with long COVID-related fatigue. The severity of symptoms seen and the impact on quality of life is profound.

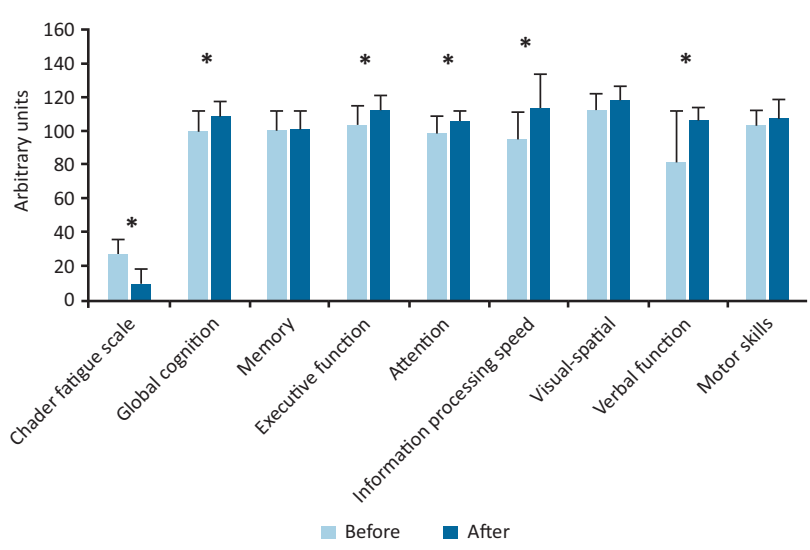

Fig 1. Scores between day 1 (before) and day 10 (after) of hyperbaric oxygen therapy. "significant difference; arbitrary units are construct specific.
Many patients are unable to work (either in manual or office-based roles), drive, participate in their usual physical activity or, at times, engage with their family in the manner they would wish. The treatment options available for such people with severe symptoms of long COVID are limited, with very few interventional options. Here, to our knowledge, we describe the first evaluated use of HBOT to manage long COVID in the UK or internationally. We report statistically and clinically significant improvements to both the overall fatigue score and a range of cognitive domains using validated scales. The effect size measures calculated are large, suggesting a substantial improvement and, thus, there is a small likelihood these results are due to chance despite the small initial sample size. These are important findings suggesting a possible positive effect of HBOT on the common long COVID-related symptoms of fatigue and 'brain fog'. These results match with the clinical and qualitative observations of patients receiving the therapy, many of whom report their lives have been transformed.

The mechanism of long COVID is still uncertain. ${ }^{1}$ One possible hypothesis is that the wide variety of changes that characterise long COVID are a result of prolonged tissue hypoxia. ${ }^{6}$ This is frequently the common denominator for many diseases that are responsive to $\mathrm{HBOT}^{31}$ Further research is needed to understand the underlying mechanisms of long COVID and positive responses seen in relation to HBOT.

While these results are important, they represent only an initial evaluation. Indeed, the sample size consists of only 10 patients. Furthermore, these patients have not been followed up for a prolonged period to assess whether the noted improvements of these long COVID-related symptoms were sustained. Based on initial informal feedback, patients do excellently in the longer term. Thus, there is a need to assess these effects of HBOT in the context of a randomised placebo-controlled prospective study. However, these initial results suggest that HBOT merits further study as a treatment option for patients presenting with long COVID symptoms (such as fatigue). Given the scale of the emerging long COVID public health emergency globally, and the still ongoing COVID-19 pandemic, there is an urgent need for larger-scale randomised placebo-controlled trials to evaluate the potential impact of HBOT in the context of long COVID. In addition, creation of a registry of patients receiving HBOT for long COVID symptoms (such as fatigue) in order to obtain followup data over time is also suggested. These are both elements 
of work currently being developed collaboratively between the Midlands Diving Chamber and University Hospitals Coventry and Warwickshire NHS Trust.

\section{Supplementary material}

Additional supplementary material may be found in the online version of this article at www.rcpjournals.org/clinmedicine: S1 - Descriptive participant level and overall group data, day 1 vs day 10.

\section{References}

1 Marshall M. The four most urgent questions about long COVID. Nature 2021;594:168-70.

2 Raveendran A, Jayadevan R, Sashidharan S. Long COVID: an overview. Diabetes Metab Syndr 2021;15:869-75.

3 Venkatesan P. NICE guideline on long COVID. Lancet Respir Med 2021;9:129.

4 Rando HM, Bennett TD, Byrd JB et al. Challenges in defining Long COVID: Striking differences across literature, Electronic Health Records, and patient-reported information. medRxiv 2021:2021.03.20.21253896.

5 Alwan NA, Johnson L. Defining long COVID: Going back to the start. Med (N Y) 2021;2:501-4.

6 Nabavi N. Long covid: How to define it and how to manage it. BM] 2020:370:m3489.

7 Mandal S, Barnett J, Brill SE et al. 'Long-COVID': a cross-sectional study of persisting symptoms, biomarker and imaging abnormalities following hospitalisation for COVID-19. Thorax 2021;76:396-8.

8 Cabrera Martimbianco AL, Pacheco RL, Bagattini ÂM, Riera R. Frequency, signs and symptoms, and criteria adopted for long COVID: a systematic review. Int J Clin Pract 2021;75:e14357.

9 Wostyn P. COVID-19 and chronic fatigue syndrome: Is the worst yet to come? Med Hypotheses 2021;146:110469.

10 Morley JE. COVID-19 - the long road to recovery. J Nutr Health Aging 2020;24:917-9.

11 Boldrini M, Canoll PD, Klein RS. How COVID-19 Affects the Brain. JAMA Psychiatry 2021;78:682-3.

12 Maxwell E. Living with Covid19. National Institute for Health Research, 2020.

13 Shah W, Hillman T, Playford ED, Hishmeh L. Managing the long term effects of covid-19: summary of NICE, SIGN, and RCGP rapid guideline. BMJ 2021;372:n136.

14 Gaber T. Assessment and management of post-COVID fatigue. Progress in Neurology and Psychiatry 2021;25:36-9.

15 Palmer S]. Government funding for research into long COVID. British Journal of Cardiac Nursing 2021;16:1-3.
16 Wong TL, Weitzer DJ. Long COVID and Myalgic Encephalomyelitis/ Chronic Fatigue Syndrome (ME/CFS) — A Systemic Review and Comparison of Clinical Presentation and Symptomatology. Medicina 2021:57:418.

17 Kirby JP, Snyder J, Schuerer DJ, Peters JS, Bochicchio GV. Essentials of hyperbaric oxygen therapy: 2019 review. Mo Med 2019;116:176.

18 Moon R. Hyperbaric oxygen therapy indications. Best Publishing Company, 2019.

19 Akarsu S, Tekin L, Ay H et al. The efficacy of hyperbaric oxygen therapy in the management of chronic fatigue syndrome. Undersea Hyperb Med 2013;40:197-200.

20 Hoof EV, Coomans D, Becker PD et al. Hyperbaric therapy in chronic fatigue syndrome. Journal of Chronic Fatigue Syndrome 2003;11:37-49.

21 Jackson C. The Chalder fatigue scale (CFQ 11). Occup Med (Lond) 2015:65:86

22 Doniger GM. NeuroTraxcomputerized cognitive tests: Test descriptions. Medina: NeuroTrax, 2013:1-16.

23 Robbins T, Keung SNLC, Sankar S, Randeva H, Arvanitis TN. Application of standardised effect sizes to hospital discharge outcomes for people with diabetes. BMC Medical Informatics and Decision Making 2020;20:1-6.

24 Eyre EL, Clark CC, Tallis ] et al. The effects of combined movement and storytelling intervention on motor skills in South Asian and White children aged 5-6 years living in the United Kingdom. Int J Environ Res Public Health 2020;17:3391.

25 Marsman M, Wagenmakers E-J. Bayesian benefits with JASP. European Journal of Developmental Psychology 2017;14:545-55.

26 Wagenmakers E-J, Marsman M, Jamil T et al. Bayesian inference for psychology. Part I: Theoretical advantages and practical ramifications. Psychon Bull Rev 2018;25:35-57.

27 Wagenmakers E-J, Love J, Marsman M et al. Bayesian inference for psychology. Part II: Example applications with JASP. Psychon Bull Rev 2018;25:58-76.

28 Wasserstein RL, Lazar NA. The ASA statement on p-values: context, process, and purpose. The American Statistician 2016;70:129-133.

29 Amrhein V, Greenland S, McShane B. Scientists rise up against statistical significance. Nature 2019;567:305-7.

30 R Core Team. R: A language and environment for statistical computing. R, 2013.

31 Choudhury R. Hypoxia and hyperbaric oxygen therapy: a review. Int J Gen Med 2018:11:431-42.

Address for correspondence: Dr Timothy Robbins, University Hospitals Coventry and Warwickshire NHS Trust, Clifford

Bridge Road, Coventry CV2 2DX, UK.

Email: timothy.robbins@nhs.net 